\title{
Article
}

\section{Music of the night: Performance practitioner considerations for enhancement work in music}

Pecen, E., Collins, D., and MacNamara, Á.

Available at https://clok.uclan.ac.uk/15459/

Pecen, E., Collins, D., orcid iconORCID: 0000-0002-7601-0454 and MacNamara, Á. orcid iconORCID: 0000-0002-8110-6784 (2016) Music of the night: Performance practitioner considerations for enhancement work in music. Sport, Exercise and Performance Psychology, 5 (4). pp. 377-395. ISSN 2157-3905

It is advisable to refer to the publisher's version if you intend to cite from the work. http://dx.doi.org/10.1037/spy0000067

For more information about UCLan's research in this area go to http://www.uclan.ac.uk/researchgroups/ and search for < name of research Group>.

For information about Research generally at UCLan please go to http://www.uclan.ac.uk/research/

All outputs in CLoK are protected by Intellectual Property Rights law, including Copyright law. Copyright, IPR and Moral Rights for the works on this site are retained by the individual authors and/or other copyright owners. Terms and conditions for use of this material are defined in the policies page.

\section{CLoK}

Central Lancashire online Knowledge www.clok.uclan.ac.uk

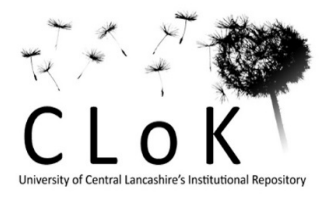


1

2

3

4

5

6

7 Music of the Night: Performance Practitioner Considerations for Enhancement Work in Music

8

9

10

11

12

13

14

15

16

17 Journal Section: Performance Psychology

18 Corresponding Author

19 Ellis Pecen, School of Sport and Wellbeing

20 University of Central Lancashire, Preston, United Kingdom, PR1 2HE.

21 E-mail: epecen@uclan.ac.uk

22
Ellis Pecen ${ }^{1}$, Dave Collins ${ }^{1.2}$ and Áine MacNamara ${ }^{1}$

2: Grey Matters for Performance Ltd. 
8 which appropriate support structures are often lacking. Accordingly, this paper aims to contribute

9 to effective interdisciplinary communication by reviewing a selection of common socio-cultural

10 and systemic issues in music performance that might broaden practitioners' contextual

11 knowledge of musicians. While this paper is not meant to be a comprehensive review of all

12 available research, we have included ample references to direct readers towards relevant material

13 on the topics being discussed. In addition, we propose that practitioners who are interested in

14 working with musicians apply a positive, culturally-sensitive, evidence-based and holistic

15 approach when translating and communicating psychology principles to musicians. We provide

16 suggestions as to how this might be achieved and also emphasize the importance of exploring a

17 range of methods, prioritizing practicality and introducing performance enhancement training

18 accurately. In doing so, we suggest that practitioners avoid focusing solely on performer

19 wellbeing, theoretical delivery formats or limited psychological skills strategies. 
11 impact, both of which ultimately affect intervention success (Martindale \& Collins, 2005).

12 Musician/performer-researchers have highlighted the positive effects of sport psychology on

13 music performance (e.g., Bellon, 2006; Hawkes, 2015; Olevsky, 2012; Thomson, 2014). Yet,

14 while awareness of psychological interventions may exist, tuition in and knowledge of how to

15 deploy performance-enhancing skills is often missing (e.g., Byo \& Cassidy, 2008; Hays 2002).

16 Notably, music is at an earlier stage of development in its research, education, practice and

17 performer acceptance of performance science (Hays, 2012) and does not yet have a robust

18 applied literature base to draw from. Consequently, professional training in performance

19 psychology coaching for music performance is a novel development. Thus, in the current

20 shortage of music specialists, psychologists from other disciplines such as sport may be the most

21 appropriate source for performance enhancement.

22 Such a decision, although convenient, may hold some problems however. An understanding of 23 domain-specific challenges in their cultural context is often overlooked, yet is key to effectively 


\section{Running Head: ENHANCEMENT WORK IN MUSIC}

1 communicating performance psychology, especially to a domain that is new to the concept (cf.

2 Willmott \& Collins, 2015). Despite the many convergences that make sport psychology

3 applicable to music performance, specific divergences exist that should be considered to

4 optimize existing methods and facilitate effective knowledge transfer (Hays, 2012; Nordin-Bates,

5 2012).

6 Reflecting these concerns, we will firstly outline the prevalence and specifics of psychological

7 and physical problems in music, while also exploring musicians' cultural milieu. Next, we

8 explore the impact of inadequate developmental support and coaching structures - factors which

9 also differentiate musicians from athletes and which generate further challenges for intervention

10 design and deployment. Finally, we discuss the implications of these considerations for

11 performance psychology training for musicians. We propose an evidence-based and culturally-

12 informed holistic approach, for which suggestions and examples will be presented.

13

14

\section{Psychological and Physical Challenges}

16 Performance demands in music and sports share common ground. For instance, extreme

17 commitment, early specialization, social isolation, training volume, identity foreclosure,

18 coaching relationships, burnout, injury, psychological pressure, expertise, talent, deliberate

19 practice, motivation, flow, perfectionism, and stigmas of psychological interventions occur in

20 both domains (Hays, 2002; Nordin-Bates, 2012). In addition, aspects often considered "unique"

21 to performing arts such as creativity, emotional expression and communicative audience

22 relationships can also be present in aesthetic sport disciplines. While athletes and musicians are

23 likely to suffer comparable challenges, however, there are distinguishing characteristics in 
1 musicians and their environments that deserve attention. Notably, challenges are in part enabled

2 by cultural beliefs and customs that exist in musical learning and performance milieus.

3 Music is rife with instances of psychological and physical issues that are currently not widely

4 and appropriately addressed in training (e.g., Chesky, Dawson \& Manchester 2004; Weller,

5 2010). For instance, prevalent conditions include psychopathology, injury (particularly overuse;

6 Kenny \& Ackerman, 2009) and poor health habits (e.g., Panebianco-Warrens, Fletcher \& Kreutz,

7 2014). Musicians also appear reluctant to seek professional help, preferring advice from peers

8 and teachers regarding matters beyond their expertise (Williamon \& Thompson, 2006).

9 Furthermore, incidences of mood and anxiety-related disorders are high, of which the most

10 commonly reported is Music Performance Anxiety (MPA - for a review, see Kenny, 2011).

11 Music task demands may add to the perceived pressure and exacerbate MPA (Hays \& Brown,

12 2004). These demands often require a combination of temporally precise fine motor execution,

13 memorization of pre-determined, lengthy and complex material, and the effective

14 communication of technical proficiency with novel musical insight. In addition, possible

15 differences in application of psychological skills (Nordin-Bates, 2012), coping (Poczwardowski

16 \& Conroy, 2002; Wolfe, 1999), personality (Kemp 1996), goals (Hays \& Brown, 2004; Lacaille,

17 Whipple \& Koestner, 2005; Lacaille, Koestner \& Gaudreau, 2007), perceptions of anxiety

18 (Papageorgi, Creech \& Welsh 2013; Gill, Murphy \& Rickert, 2006), psychopathology (Mula \&

19 Trimble, 2009), identity (e.g., Ivaldi \& O'Neill, 2001), confidence (Sinden 1999), learning

20 cultures (Burt-Perkins, 2013) and systemic differences impact the occurrence of common issues

21 and further distinguish music from sport.

22 For instance, available literature does not reflect a robust sense of confidence and control in 
1 musicians, who typically appear low in confidence and high in anxiety (e.g., Nordin-Bates, 2012;

2 Sinden, 1999; Talbot-Honeck \& Orlick, 1998). Views of performance "depending on the day" or

3 "nerves being outside of their control" are not uncommon (e.g., Ivaldi \& O'Neill, 2000).

4 Cognitive factors that may raise anxiety levels include one's reaction to lack of control,

5 unrealistic perfectionism and perceptions of low probability of success (Kenny, 2011; Lehrer

6 1987). As limited training exists (Osborne, Kenny \& Cooksey, 2007; Osborne, 2014; Patson \&

7 Waters, 2015) for teachers or students to appropriately address such issues, MPA is typically

8 accepted and quietly left unresolved (Wilkinson, 2005). Although research has started to address

9 perfectionism in early music training (Patson \& Osborne, 2015), unrealistically perfectionistic

10 strivings may often be present. These might be exacerbated by digitally perfected recordings that

11 leave performers and audiences alike with physically unattainable mental representation of

12 pieces (Hays \& Brown, 2004; Kruse-Weber \& Parncutt, 2014).

13 As MPA appears widespread, it has been researched extensively (see Kenny 2011) yet

14 predominantly through a pathological rather than a positive psychological lens (Nordin-Bates,

15 2012). Thus, research has mainly produced intervention studies aimed at ameliorating symptoms

16 of MPA rather than positive performance enhancement. Of concern, and despite many positive

17 outcomes of these interventions (Kenny, 2011), it seems musicians are not using psychological

18 strategies as their primary coping source. However, risky coping methods such as beta-blockers

19 and alcohol are commonly reported (e.g., Chesky \& Hipple 1999; Fishbein et al., 1988; Steptoe,

20 1989). Indeed, use of beta-blockers is even occasionally promoted by leaders (Tindall, 2004). In

21 addition, such drugs are passed around without prescriptions and are easily obtained in music

22 environments (Dunkel, 1990). 
1 The topic of beta-blockers is controversial in music and may often divide opinion. It is beyond

2 the scope of this article to delve deeply into all facets of the matter, however, it is worth pointing

3 out some considerations for practitioners. Naturally, there may be considerable differences

4 between the type of substances used and musicians' circumstances and motives for doing so.

5 However, it is worth pointing out that potentially harmful performance enhancing drugs (PEDs)

6 are freely circulated in musical settings and that this has repercussions. This posits several

7 questions worthy of consideration. Might drug-use not negatively affect performer health?

8 Should drugs such as beta-blockers continue to be considered "acceptable" despite healthier

9 psychological treatment options being available? Although beta-blockers might seem to enhance

10 technical performance, they might also give rise to lackluster performance. Are beta-blockers

11 then worth the risk? Are they even in line with the purpose of music if they can dampen the

12 energetic buzz of a performance? Are the ethics observed in music towards the use of beta-

13 blockers justifiable? Does music's acceptance towards beta-blockers cause healthier methods to

14 take a backseat in favor of "quick fixes"? How does knowing you owe a performance to a drug

15 affect feelings of self-efficacy and cognitive reconditioning? Particularly at risk are musicians

16 who are not taking such drugs under medical supervision. Unfortunately, this may well be due to

17 the lack of appropriate introduction and instruction on how they can use healthier coping

18 methods such as psychological strategies, exercise and healthy lifestyle habits.

19 Although covert drug use (total across performance and social) might be comparable between

20 musicians and athletes, music's lenient attitude towards PEDs stands in contrast to the attitude

21 strived for in sports (e.g., Douglas 2007). In sports, PED use is heavily sanctioned by society,

22 media and leaders in the field. In musical environments, however, attitudes towards PEDs appear 
1 lax, duty of care is neglected and dysfunctionality is sometimes even glorified within the

2 industry (e.g., Novick \& Steen, 2014). Thus, practitioners are likely to be faced with an anxiety-

3 ridden culture that is also lenient towards the use of performance enhancing substances - two

4 aspects that will likely impact content and conduct of the intervention.

5 Finally, and despite the number of successful intervention studies in the literature, practical

6 training in music performance enhancement techniques is somewhat sparse. Initiatives are

7 developing globally (e.g., Chesky, et al., 2004; Liertz, 2007) yet even more proactive and

8 integrated approaches are called for by researchers and students alike (e.g., Atkins, 2009;

9 Parncutt, 2007; Weller, 2010). Furthermore, although a growing body of research demonstrates

10 the benefits of performance psychology in music (e.g., Braden et al., 2015; Osborne, 2013;

11 Osborne, Greene \& Immel, 2014), institutions appear reserved towards change. Teachers appear

12 reluctant to incorporate science, and academic work is reduced to allow as much time as possible

13 for practice (Parncutt \& Williamon, 2005; Weller, 2010) as performers often consider everything

14 that is not practice as "a waste of time" (Brown, 2012). Consequently, initiatives, if available,

15 can often be mostly theoretical and lacking in practicality and systematic delivery (e.g., lecture

16 or workshop formats are typical - Weller, 2008). Although clearly well-intentioned, such

17 theoretical formats appear to lack traction with student-performers (Brown, 2012) and do not

18 accurately reflect performance psychology training. Indeed, structured, systematic and

19 comprehensively interdisciplinary training that practically communicates the tools necessary to

20 optimize physical and psychological functioning (rest, hydration, nutrition, conditioning,

21 psychological skills, etc.) is uncommon.

22 Consequently, at least in the environments with which we are familiar, performance science is 
1 often considered an academic subject with little direct applicability. These limited views are,

2 perhaps, understandable given the common lack of implementation. However, they also pose an

3 additional challenge to practitioners who will need to appropriately introduce to an audience that

4 is new to performance psychology.

5 In continuation of the previous point regarding the pathological focus of music psychology

6 research, it is worth considering the limited availability of research focusing on psychology for

7 performance. The latter focus distinction is made to differentiate between the 3 evolutionary

8 stages of a support science (Collins \& Kamin, 2012); namely, psychology 1) through, 2) of, and

9 3) for, performance. The first two stages are primarily concerned with generating scientific

10 publications relating to aspects of the parent (1) or a subject-specific (2) sub-discipline rather

11 than focusing on the implications of applied performance science and producing findings that are

12 directly applicable to performers (Winter \& Collins, 2015). As evolution through these stages is

13 approximately sequential, the field of music performance research would appear to be only

14 recently progressing onto the third phase. Combined with the comparative lack of fine motor

15 control research essential for music (Collins, 2013), practitioners have little practical musician-

16 specific research to draw from. They will also need to understand that limitations present in

17 music performance environment are also due to music being at an earlier evolutionary stage in

18 which practical research and its applied practice are not yet the established norm. Thus,

19 demonstrating the field-applicability of performance psychology within practical constraints

20 while taking into consideration attitudes towards key constructs (e.g., anxiety and substance use)

21 may be an essential component to successful, especially institution-based, interventions.

\section{Beliefs as Barriers}


1 Task demands, inadequate support structures, limited research and biopsychosocial

2 interactions (relating to biochemical, physiological, psychological, social and cultural factors;

3 Ray 2004) impact the occurrence of the aforementioned problems. Yet problems are also rooted

4 in cultural norms that have ignored scientific advances and upheld questionable beliefs regarding

5 talent and practice (e.g., Hays, 2012; Weller, 2008). Cultural beliefs might impact musicians'

6 willingness to engage with more adaptive means of practice and performance preparation and

7 execution. This occurrence is not unique to music and can also be observed in subcultures of

8 sports (e.g., Wilmott \& Collins 2015).

9 For instance, MPA appears to be perceived as ubiquitous, inevitable and not simple to control

10 (Gill, Murphy \& Rickert, 2006; Hays 2002; Ivaldi \& O’Neill, 2001). As appropriate support

11 structures are perceived lacking, such perceptions may underpin common dysfunctional

12 behaviors. They might, for example, contribute towards disbelief in performance psychology as

13 the concept of gaining control over performance might seem novel. Similarly, the stereotype of

14 the tormented artist and "suffering for one's art" is common. This not only encourages

15 dysfunctional behaviors but also prevents musicians from seeking help (Quarrier 1993). Indeed,

16 science itself may seem incompatible with artistry and be eschewed (Hays, 2012). Additional

17 barriers may be caused by beliefs that "a talented person learns by just doing it", that "music is

18 ethereal and unquantifiable", that "the current tradition is successful due to technical

19 advancements in playing as compared to century X" and that examples exist of "experts who

20 have not used science to become excellent." Similarly to sports ("X does it so therefore so should

21 I"; Collins, 2014), role models may be used to justify behaviors.

22 As mentioned earlier, musicians are not societally expected to be excellent role models and 
1 their potential 'artistic' dysfunctionality is even occasionally glorified (e.g., Novick \& Steen,

2 2014). Thus, surmounting the barriers created by role models who dispatch ill-considered advice

3 or who may even be unaware of the implicit knowledge they possess and strategies they actually

4 deploy, poses an additional challenge. Despite this, experts who openly discuss the deployment

5 of (sport) psychology (e.g., "Pianist tells of adding sports psychology to his repertoire", 2014)

6 and more efficient practice habits (e.g., Auer, 2003; Davis, 2005; Leinsdorf, 1999) do exist.

7 However, such behaviors do not seem common practice as beliefs in talent and practice volume

8 persist. Given the recency of relevant research in this area (e.g., Osborne, 2013; Osborne et al.,

9 2014) the concept that psychology might serve to achieve one's own maximum performance

10 potential has yet to enter the culture. Appropriate communication and demonstration of how

11 performance psychology fits into and supports this spontaneous and artistic image of music

12 making is therefore key to impact.

Developmental Support and Coaching Structures

14 Cultural beliefs are reflected at various hierarchical levels in music education. The current

15 lack of appropriate developmental support and coaching structures is, in part, encouraged by

16 conservative beliefs held in education, even in the presence of potential practical and financial

17 constraints. Music institutions play a pivotal role in development as musicians typically study

18 formally from an early age until young adulthood. Hence, such establishments are a key location

19 to introduce performance science to musicians. It is therefore relevant for practitioners to not

20 only understand the institutional impact on belief systems and attitudes, but also issues in the

21 institutional structures which may inform potential future collaborations. For instance, major

22 barriers to progress in musical development are affected by a) the belief that musical excellence 
1 results from inherent talent coupled with practice volume, b) the lack of training for teachers and

2 c) disregard for talent development. We will subsequently review these key aspects.

\section{Talent Identification}

4 The belief in inherent talent is enshrined in music's selection and examination procedures,

5 which are, in turn, based on presented merit and a momentary demonstration of performance

6 achievement (Bennet \& Stanberg, 2006). Unfortunately, such performance "snapshots" cannot

7 reliably predict future performance (MacNamara \& Collins, 2009). Thus, the focus remains

8 solely on talent identification and performance skill, with little consideration for developmental

9 potential (Carey, 2010). Reflecting this belief, the importance of talent development (TD) has not

10 received the same attention as it has in sports and appears for many to be a novel concept.

11 Instead, a musician is expected to "be talented", absorb information regarding technique and

12 musicality during music lessons and autonomously engage in unsupervised practice (Evans,

13 2015; Miksza, 2011; 2015; Quarrier, 2013). Content and systematic delivery of lessons may also

14 be highly varied, unstructured and contradictory (Baughman 2014; Gaunt, 2007) as "performer-

15 teachers" (Huhtanen, 2004; Mills, 2006) fit teaching commitments around their own

16 performance career schedules. As such, conservatoires could appear to prioritize maintaining

17 themselves and their prestigious staff (Carey, 2010) over maximizing student development.

18 Notably, talent conversion markers (i.e., what percentage of entrants actually make it - an

19 increasingly common outcome measure in sport) are nowhere to be seen.

20 Practice

21 Similar to sports, beliefs in high practice volume persist. Yet, as adequate knowledge of

22 optimal lifestyle and training habits to support such intense activity is missing and practice is 
1 unsupervised, striving for high practice volume may carry particularly detrimental consequences.

2 As music education and psychology research point out (Bonneville-Roussy \& Bouffard, 2015;

3 Evans, 2015; Mikza, 2011; 2015), exploring ways of increasing practice quality in music is a

4 crucial need. Musicians' "more is better" notion (Quarrier, 2013) seems unfounded as practice

5 gains were found to decrease after two hours with little additional benefit after four (Welford,

6 1968). Of course, relating not to mere quantity spent practicing but rather, to qualitative

7 differences in practice activities, deliberate practice can predict outcome (Duke, Simmons \&

8 Cash, 2009; Platz, Kopiez, Lehmann \& Wolf, 2014; Williamon \& Valentine, 2000). For instance,

9 research shows that components such as self-regulation, self-efficacy, planning, motivation,

10 effort and evaluation impact deliberate practice quality in music (Bonneville-Roussy \& Bouffard,

11 2015; Ericsson, Krampe \& Tesch-Romer, 1993). However, the specifics of what exactly

12 constitutes optimal music practice have not been as thoroughly researched in music as in sports.

13 Only total duration estimates exist, which have included suboptimal practice behaviors rather

14 than actual duration of qualitative activities linked to deliberate practice (Platz et al., 2014).

15 Thus, existing positive correlations between practice quantity and performance outcome are

16 questionable. Hence, practitioners will have little domain-specific literature to draw from and

17 will likely have to explore ways of improving practice content while working with musicians'

18 inclinations towards high volume practice. Doing so is especially relevant as optimal practice is

19 not always discussed in lessons (e.g., Kostka, 2002). As mentioned earlier, some students may be

20 aware of efficient practice behaviors, but may not know how to deploy these skills (see Byo \&

21 Cassidy, 2008; Miksza 2011; 2015).

\section{Coaching Structures}


1 In music education there is an apparent tendency to focus on technique and musicality rather

2 than the specifics of practice content and performance preparation (Zenker, 2004). In increasing

3 contrast to sports, teachers are appointed on the basis of performer prestige and are not

4 necessarily trained in pedagogy, nor do they have coaching-style support structures available to

5 them (Carey \& Grant, 2014). This leads to the common problem of experience-based tuition,

6 which draws mostly from the teacher's instrument-specific knowledge. This often leads to

7 "teaching as the teacher was taught" (Carey \& Grant, 2014). Such "pedagogical inertia"

8 (Schulman, 2005) continues, allowing outdated principles to be passed on and scientific

9 advances to be ignored.

10 The lack of pedagogical training may also complicate teachers' ability to explicitly

11 communicate implicit or tacit knowledge they might be unaware of. The latter issue has also

12 been explored in sports coaching (e.g., Nash \& Collins, 2006). It is equally relevant to music as

13 this lack of awareness of knowledge might impede teachers from communicating what it was

14 exactly that they did that lead to their excellence. Together with beliefs in inherent unquantifiable

15 talent, this may prevent expert knowledge from being disclosed to students. This is relevant to

16 practitioners as they may be the ones to communicate the characteristics necessary to achieve

17 excellence in music but will have to do so in harmony with advice given by the revered teacher.

18 The latter point should be considered as conservatoire tuition follows the authoritative master-

19 disciple model in which the teacher holds a position of power and the student is a passive

20 recipient of knowledge (Carey \& Grant, 2014). This model is comparable to authoritative, coach-

21 athlete relationships still observed, but also increasingly questioned, in sports cultures. It also

22 poses similar issues such as loyalty to the coach and the boundaries of the relationship (e.g., 
1 Burke, 2001). Such a model can be both positive and negative to aspects of performance (Carey

$2 \&$ Grant, 2014).

3

4

5

6

8 practitioner. Thus, substantial individual differences may exist between musicians regarding their

9 knowledge of psychology. Likewise, variations may exist in the settings practitioners are

10 required to work in (e.g., private sessions, compulsory sessions as part of a curriculum, general

11 workshops at orchestras etc.). Hence, practitioners should also be prepared for musicians who

12 may have reservations towards interventions originating from another domain (e.g., Hays, 2012;

13 Hawkes, 2015). Therefore, based on the information we have reviewed thus far, we suggest that

14 practitioners deploy a culturally-sensitive and holistic approach that 1) considers the impact of

15 domain-specific challenges and divergences, 2) constantly explores how to communicate

16 information in a culturally appropriate manner, 3) effectively demonstrates what performance

17 psychology training entails for musicians, and, 4) explores the deployment and optimization of a

18 wide range of available methods. These include lifestyle habits and the development of key

19 psychological characteristics. In support of this stance, we will subsequently offer suggestions as

20 to how this might be achieved.

\section{Introducing Performance Psychology}

22 Dialogue between music researchers and teachers is key yet not common (Renshaw, 2004).

23 Consequently, many performers have an incorrect idea of what performance psychology training 
1 entails. Hence, a key task for practitioners might include introducing performance psychology

2 accurately and demonstrating its field-applicability in a culturally appropriate manner. This is

3 relevant as it appears that the term "music performance psychology" is used inconsistently,

4 referring to theoretical research, coaching and counseling-based initiatives. Hence, no accurate

5 reflection of what goes on in performance psychology training, as it is known in sports, is

6 offered. Thus, demonstrating that it is neither "talk therapy for troubled musicians" nor

7 "academic" is crucial. To achieve this, examples of step-by-step, practical approaches that might

8 be used to inform interventions for music performance enhancement have been offered by

9 several authors, including Brandon and Ivans, (2009) and Greene (2012a). Both training

10 paradigms have been explored by Osborne et al. (2014) and Braden et al. (2015) respectively.

11 A key point to explain, and a way of phrasing it, is perhaps to communicate that performance

12 psychology serves to assist musicians in executing their musical skills successfully under high

13 stress conditions. It might also be useful to point out that performance science does not seek to

14 alter musicians' artistry but rather, to support it, allowing their art to be communicated with as

15 little interference from negative effects as possible. Musicians need to know that their technique

16 and musicality will not be harmed and that conventional music lessons can co-exist in harmony

17 with performance psychology training.

18 Considering Cultural Challenges

19

20

21

22 Understanding music's learning culture, milieu, language and systemic hierarchy can render

23 credibility and flexibility to the practitioner. Furthermore, musicians appreciate empathic

24 practitioners who individualize their approaches and have domain-specific knowledge (Guptill, 
1 Zaza \& Paul, 2000; Hays 2002). A key component to successful and culturally-sensitive

2 communication is the appropriate use of terminology. The importance of word choice is not only

3 important for effective knowledge transfer. For instance, literature on pre-performance priming

4 shows that words may also have a psychological impact that can improve or deteriorate

5 performance outcome (e.g., Ashford \& Jackson, 2010). It is therefore important to avoid phrasing

6 that might invoke negative associations in musicians.

7 In aesthetic performance disciplines especially, the artistic identity is central. For instance,

8 even though musicians are "small muscle athletes" (Quarrier, 2013), similarities with athletes

9 may not seem compatible with musicians' worldview (Hays 2002; 2012). Accordingly,

10 terminology should be used appropriately and contextual intelligence should be maintained

11 (Hays 2002; 2012). This might avoid invoking aversion to "sport-based methods", which might

12 be viewed as irrelevant or even a threat to musicians' artistic identity and interpretations. Beliefs

13 such as "if I become mentally tough I might become less emotional in my music" or "if I

14 exercise I might get a bulky physique and no longer look like an artist" may circulate. Hence,

15 care should be taken not to encourage these misconceptions through poor phrasing and choice of

16 terms. Also, labeling skills with terminology such as "periodization" might not resonate well

17 with musicians as the culture has not yet been introduced to such terms and musicians might feel

18 subjected to a "training protocol for athletes". Although periodization might be used similarly in

19 music as in sports, it might for instance be introduced as a long-term planning method for

20 maximal skill development and performance preparation, thereby explaining the principles in

21 terms that artists can relate to. This could also be achieved by term exchange; using "resilience"

22 instead of "toughness" (Hays, 2012; Osborne, 2013), "flow" instead of "in the zone" and 
1 avoiding terms such as "enhancement" which might not fit the purpose of art (Nordin-Bates,

2 2012). Similarly, phrasing as "be strong for your art" rather than "toughen up", "be disciplined to

3 do X for the sake of your art despite feeling Y" or "do what it is right for your music, not what is

4 easy for you", may better capture the essence of how psychology can support music without

5 posing a threat to artistic pursuits and identity.

6 Working with the culture.

8 Working with potentially engrained socio-cultural beliefs in any domain can pose specific

9 challenges that require modification of content and delivery of existing interventions. Therefore,

10 exploring ways of introducing gradual, incremental change in congruence with the existing

11 norms might be most suitable (Weller, 2004). When offering solutions to musicians, it is worth

12 considering the position of the respected teacher and the tradition that is deemed successful

13 (Carey, 2010; Parncutt \& Williamon, 2005). Rather than discounting existing teacher advice,

14 alternative approaches might best be introduced carefully to allow performance psychology to

15 co-exist in harmony with existing music lessons.

16 Also within this context, phrasing in congruence with socio-cultural beliefs and self-schemata

17 may be impactful. For instance, "forget talent, let's focus on enhancement" might resonate less

18 well than "there are things musicians can do to make their skills better regardless of how talented

19 they are". Or "quit drug use now" might be communicated as "we know strategies that have

20 produced the same results as drugs and might lead to even better performance" or "let us explore

21 ways to make the success of your performance attributable to you rather than a pharmaceutical

22 drug". Another option is using existing beliefs to your advantage. For instance, "if an artist must

23 suffer then why not suffer adaptively?" - the argument being that intense exercise, planning and 
1 discipline for the sake of your art can surely induce some "suffering" as well? Similarly,

2 changing habits feels uncomfortable, yet "might a true artist not be willing to do anything to

3 improve one's art and performance?" An additional approach might be to integrate positive

4 characteristics associated with artists such as open-mindedness and versatility; for example,

5 "should an artist not be open-minded and draw from as much useful knowledge as possible,

6 regardless of where this knowledge comes from?"

$7 \quad$ Musicians (like some athletes) might use examples of role models who did not receive

8 performance psychology training to question the validity of the intervention. In this instance,

9 practitioners might point out that there is research documenting how expert musicians make use

10 of advanced strategies and health behaviors, albeit occasionally without being explicitly aware of

11 it and labeling their activities in scientific terms (e.g., Bellon, 2006; Talbot-Honeck \& Orlick,

12 1998). For instance, highly skilled musicians employ different psychological strategies such as

13 planning, self-regulation and evaluation than average musicians (Araujo, 2015). Experts also

14 exhibit more knowledge of health responsibility than music students (Rickert, Barrett \&

15 Ackermann, 2015). Such arguments might be used to demonstrate to performers that examples of

16 negative role models should not be used to justify dysfunctional behaviors. Simultaneously, they

17 may also point out how performance psychology can be used to analyze and teach the

18 characteristics that typify elite performers.

19 Another aspect worthy of consideration, especially given the high injury rates, is the striving

20 for high practice volume. As it is an engrained part of the culture, a gradual approach that

21 explores more efficient and effective ways of skill development by improving practice content

22 might be beneficial. This might be achieved via using random, mental, or combination practice 
1 and increasing variation, self-regulation, conditioning and focus (see Wulf \& Mornell, 2008). Of

2 course, deliberate practice (DP) should be prioritized over "mindless repetitive practice", which

3 can be sustained for long periods of time. Musicians may not have been explicitly instructed on

4 how to make use of DP in their training. Practitioners could therefore beneficially teach the

5 characteristics of DP (intentional, repetitious, focused on performance improvement, designed

6 according to the performer's current skill level, combined with immediate feedback and not

7 inherently enjoyable). Such quality of practice, underpinned by appropriate environmental

8 support, motivation and effort, is the crucial determinant of expertise (Ericsson et al., 1993).

9 Such options for increasing quality might be better received than sudden reductions in duration.

\section{Considering divergences.}

11 Fine motor control.

12 In contrast to sport disciplines that involve untimed continuous movements, music

13 performance consists of discrete rhythmic actions that adhere to regular cycles of timed events

14 (Janzen, Thompson, Ammirante \& Ranvaud, 2014). Furthermore, such discrete motor actions are

15 often maintained over long periods of time as concerts may last for hours. Thus, musicians may

16 often continuously exert fine muscles over long durations of time. Although sport science has a

17 robust research base in exploring gross motor action, research on fine motor control is

18 comparatively lacking (Collins, 2013). The difference between the execution of fine and gross

19 motor tasks and its implications should be considered.

20 Available literature shows a high incidence of upper body injuries in musicians (e.g., Bejjani,

21 Kaye \& Benham, 1996). Consequently, musicians may have an increased sensitivity in the body

22 parts they use for playing (Chan \& Ackermann, 2014; Watson, 2009, p. 74). This might carry 
1 implications on several levels. For instance, the fear of hurting the body in the slightest may

2 impact musicians' engagement in physical activity, even if this may offer considerable

3 prophylactic and health benefits. This might best be considered when recommending exercise

4 regimens or conditioning exercises that require use of musicians' hands and fingers. Therefore,

5 careful, gradual introduction to such concepts may be necessary to avoid injury (e.g., Chan \&

6 Ackermann, 2014). This is also relevant as musicians' overused body parts may respond heavily

7 to the smallest, seemingly insignificant additional physical load. If additional load is

8 inappropriately induced on already excessively trained tissue, the risk of injury is increased. This

9 can have detrimental effects on fine motor control, subsequently compromising psychological

10 wellbeing (Fry, 1986; Watson, 2009).

11 Health habits.

12 In contrast to sports, the importance of physical conditioning, deployment of psychological

13 skills and adherence to health-promoting behaviors is not well-established in music. This is

14 unfortunate as many prevalent psychological and physical ailments might be ameliorated by

15 deployment of adaptive strategies and lifestyle habits (e.g., Chan \& Ackermann, 2014; Kenny \&

16 Ackermann, 2009). A major point to consider is that many musicians may not have yet realized

17 that care in these areas is not optional but essential to achieve their maximum performance

18 potential. Therefore, when communicating the importance of the latter point, adaptive behaviors

19 such as appropriate planning, exercise, nutrition and rest might best be related to their direct

20 utility for musical practice and performance. Practicality, procedural knowledge and real-world

21 application, for example in the form of performance simulation, are key (Greene, 2002;

22 Williamon, Aufegger \& Eiholzer, 2014). For instance, instead of generally promoting 
1 cardiovascular exercise, one might point out that cardio can be used to invoke the symptoms

2 associated with performance, such as high heart rate, stress, sweat and fatigue. This creates an

3 opportunity for musicians to practice deployment of psychological skills during "a simulations"

4 of physical "performance" stress while simultaneously making their "heart and body stronger to

5 support a more unimpeded expression of their art". Such phrasing might resonate better with

6 musicians than "use combination training". In addition to increased activity, adequate recovery

7 might also be a novel addition to musicians' routines, and they might benefit from understanding

8 how inadequate recovery may lead to e.g., injury, decreased alertness and muscle fatigue and

9 ultimately result in suboptimal practice and performance. In contrast, adequate rest and sleep can

10 improve stamina and motor skill consolidation (Allen, 2013; Simmons \& Duke 2006; Simmons,

11 2012) so musicians can benefit more from the time invested in practice and increase both

12 practice and performance quality. Likewise, due to musicians' tendencies towards high practice

13 volume, any activity that is not directly perceived as traditional practice may be viewed as a

14 waste of practice time (Brown, 2012). In this instance, pointing out the relevant benefits of

15 exercise to musicians (e.g., alertness for practice, psychological discipline, stronger body to

16 support practice, stronger heart for performance, contribution to "good stage looks" etc.) could

17 help to better facilitate the communication of adaptive lifestyle habits and strategies so that these

18 are not perceived as "sport" but as performance preparation methods to support art.

19 Goals \& coping styles.

20 Musicians tend to strive towards subjective and personal goals rather than a quantifiable

21 "personal best" (Hays, 2002; Talbot-Honeck \& Orlick, 1998). Their goals tend to be intrinsic

22 (Lacaille et al., 2005; 2007) and they appear to have a tendency towards using emotion-based 
1 coping styles (Wolfe, 1990; Poczwardowskyi \& Conroy, 2002). Winning is generally not a main

2 goal unless to secure a position or future performance engagements. Instead, communication of

3 personal artistry with and pleasing of the audience is strived for (Hays, 2002; 2012).

$4 \quad$ Such subjective goals make specific goal setting complex (Hays \& Brown 2004). As

5 systematic coaching to improve on performers' specific weaknesses is often lacking, sport

6 psychologists might make use of performance profiling or goal attainment scaling to focus on

7 personal progress rather than assigning numbers to performances (Nordin-Bates, 2012). In order

8 to determine which specific skills might need improving in musicians, questionnaires such as the

9 Performance Skills Inventory (Greene, 2013) and Psychological Characteristics for Developing

10 Excellence Questionnaire (PCDEQ; MacNamara \& Collins, 2011) might also be employed.

11 Regarding coping styles, it is worth considering that emotionality has been linked to length of

12 musical training and trait emotional intelligence (e.g., Petrides, Niven \& Mouskounti, 2006).

13 While practitioners might respect that emotionality and emotion-based coping might be deeply

14 engrained in the musician and focus on strengthening preferred coping styles, the training of

15 alternative responses (e.g., problem-, appraisal-focused, use of adaptive behaviors) can help to

16 expand musicians' coping arsenal beyond emotional and maladaptive coping. A variety of coping

17 skills are necessary and the knowledge of when to use which response depending on context, is

18 paramount.

19 Memorization \& MPA.

20 Memorized performance positively affects communication with the audience (Ginsborg, 2004;

21 Williamon, 1999) -a major goal for musicians. Often material is pre-determined, lengthy and

22 complex. Therefore, anxieties related to memorization may underpin a large proportion of the 
1 prevalent MPA (Hays \& Brown, 2004; Killagh, Thompson \& Morgan, 2015). Practitioners might

2 for instance incorporate robust memorization strategies such as mental practice (Bernardi,

3 Schories, Jabusch, Colombo \& Altenmueller, 2013), structural analysis, performance cues

4 (Chaffin, Demos \& Crawford, 2009) and decision-making responses (Kruse-Weber \& Parncutt,

5 2014) to recover from and prevent lapses and build confidence (for a review of music

6 memorization strategies, see Chaffin, Logan \& Begosh, 2008; Mishra, 2004; 2011).

\section{Proposed Holistic Approach}

\section{$8 \quad$ Perceptions of arousal.}

9 Research in music has predominantly applied a pathological lens, often viewing arousal and

10 anxiety as predominantly negative and focusing on alleviation of MPA symptoms (Nordin-Bates

11 2012). Hence interactions between MPA, biopsychosocial factors and task demands have been

12 left largely unexplored (Nordin-Bates, 2012). Therefore, practitioners should take care not to

13 copy the pathological perspective from available literature but rather, adopt a more positive

14 psychological lens. They should consider the impact of biopsychosocial interactions and

15 contextual demand.

16 A debatable assumption often circulated in music is that high physical arousal may not be as

17 advantageous for musicians because fine motor control tasks do not allow for a similar

18 expression of adrenaline as gross motor tasks do (e.g., Bellon, 2006; Greene, 2002). This

19 preoccupation with arousal down-regulation is not necessarily beneficial however, as ideal

20 anxiety for optimal performance depends on the performer, level of physical exertion required to

21 play the instrument, emotionality and situational demand (Hanin, 1997; Osborne et al., 2014;

22 Wesner, Noyes \& Davis, 1990). 
1 Consequently, while relaxation strategies certainly have their place in the mental skills toolkit,

2 musicians might also benefit from exploring the entire repertoire of psychological skills and

3 physical conditioning. For instance, treating MPA as a distraction and focusing on directing

4 attention to task-relevant thoughts rather than symptoms might be a beneficial alternative

5 (Connolly \& Williamon, 2004; Nordin-Bates, 2012). Another might be to foster mentally

6 resilient attitudes towards anxiety and encourage a proactive attitude that encourages musicians

7 to distance themselves from symptoms and "transcend their body to focus solely on their art" or

8 "use the fear to their advantage". This might be useful, as a state of true relaxation is unlikely to

9 be reached (or even desirable) before performance (e.g., Greene, 2002; Hays \& Brown, 2004).

10 More recent evidence-based therapies for MPA, such as Acceptance and Commitment

11 Therapy, and performance enhancement approaches, such as Mindfulness-Acceptance-

12 Commitment (Gardner \& Moore, 2001) have adopted a more neutral perspective of anxiety.

13 These approaches encourage acceptance of anxiety symptoms and teach recipients to apply

14 flexible and adaptive behaviors. Both have shown beneficial effects on musicians and might be

15 used to inspire future interventions (Juncos \& Markman, 2015; Steyn, 2013).

16 Music performance heavily recruits small muscle and simultaneously produces high cognitive

17 load. Although musicians (as with expert sports athletes) make performance appear like an

18 effortless low impact activity, the cardiovascular demand should not be underestimated; heart

19 rates may fluctuate between 100 and $173 \mathrm{bpm}$ for durations of 20-60 minutes (Clark, Holmes,

20 Feeley \& Reffing, 2011; Morgenstern, 2005; Service, 2012). This intensity should be considered

21 as musicians who experience performance as highly intense and exerting would likely find a sole

22 focus on relaxation to be of limited use. As performance intensity varies intermittently, an option 
1 might be to explore the use of high intensity interval or resistance training and acute versus

2 steady state exercise which have shown benefits for musicians when used purposefully (e.g.,

3 Ackermann, Adams \& Marshall, 2002; Wasley, Taylor, Backx \& Williamon, 2012). Training

4 suggestions for gradually introducing musicians to physical exercise are for instance offered by

5 Taylor \& Wasley (2004).

6 Pillars of performance.

8 Given the training climate in music, the fundamentals underpinning excellence in

9 performance such as appropriate lifestyle habits, psychological characteristics, practice quality

10 and performance preparation, may be suboptimal. For instance, concepts such as resilience,

11 growth mindset, grit and self-control (Dweck, 2006; Duckworth, Petersen \& Matthews, 2007)

12 are crucial psychological characteristics needed to achieve excellence across performance

13 domains, including music (e.g., MacNamara \& Collins, 2009; Talbot-Honeck \& Orlick, 1998).

14 Key to success, especially in young developing performers, is the presence of Psychological

15 Characteristics for Developing Excellence (PCDEs) which include commitment, focus,

16 distraction control, imagery, realistic performance evaluations, quality practice, goal setting,

17 coping, planning, organizational skills, self-awareness (MacNamara, 2011) and, specific to

18 musicians, creativity, spontaneity, and flexibility (Talbot-Honeck \& Orlick, 1998). For instance,

19 self-discipline was identified by musicians as the major factor that would lead to better practice

20 habits (Byo \& Cassidy, 2008). In addition, motivation, self-regulation and self-determination

21 have shown crucial impact on practice and performance outcome (Bonneville-Roussy \&

22 Bouffard, 2015; Evans, 2015; McPherson et al., 2016; Miksza, 2011; 2015). Neglecting such

23 physical and psychological fundaments in favor of isolated strategies to remedy symptoms of 
1 MPA seems an incomplete approach to music performance enhancement and might inadequately

2 address underlying factors that contribute to its symptoms. Consequently, it is worth considering

3 that symptoms may also be a consequence of suboptimal psychological and physical skill

4 development and performance preparation. Therefore, the application of a holistic approach that

5 explores the development of performance-facilitating psychological characteristics and lifestyle

6 habits in conjunction with a range of available methods and strategies might provide a better

7 alternative to address common challenges in music. These pillars of performance are

8 fundamental and should be considered throughout the proposed holistic framework.

$9 \quad$ Possible Recommendations.

10

11 A possible strategic starting point to a more holistic approach might be to introduce long-term,

12 detailed planning akin to periodization, and encourage the identification of specific technical,

13 musical and performance goals. Training solutions to common issues such as building stamina or

14 strengthening the body to endure practice demands, could be embedded into this plan. Within

15 this context, exploring how tapering might be used to prevent injury and maximize recovery to

16 peak for a performance as well as how some expert musicians use it (e.g., Talbot-Honeck \&

17 Orlick, 1998; Thomson, 2014) can be a valuable strategy. Modifications such as reduction of

18 tapering time (e.g., days instead of weeks and the 48-hour "muscle recovery rule"; Quarrier,

19 1993) might be explored to accommodate the difference in physicality. Psychological skills

20 might be trained in conjunction with physical preparation in a similar periodized manner (see

21 Holliday et al., 2008 for practical suggestions). Periodized training programs systematically vary

22 volume and intensity to maximize performance gains. Training is organized in such a way that

23 peak performance is likely to occur at a specific time (e.g., competition). Periodized 
1 psychological training cycles can similarly progress from an education and acquisition stage of

2 psychological strategies and skills, to their practice, automation, implementation, and

3 performance (Holliday et al, 2008). This allows for psychological skills to be practiced and

4 automated by the time a crucial event occurs.

5 Similarly to sports, performance opportunities involving low perceived threat (e.g., playing in

6 informal settings) might be incorporated to gradually build towards important events and offer

7 opportunities for goal and performance progress evaluation. Music experts' use of "simulation

8 concerts" has already been documented (e.g., Talbot-Honeck \& Orlick, 1998; Williamon et al.,

9 2014). This also demonstrates that error-free learning is not necessarily advantageous and that

10 using past errors to inform and improve the next performance can help musicians realize the true

11 extent to which they are in control. This is relevant as the misconceptions that 'practice makes

12 perfect' and "good performance just happens" still circulate, which may impede musicians from

13 realizing which factors contribute to performance success and how they might gain control over

14 these.

15 Accordingly, an emphasis on building robust self-confidence and self-efficacy as in sports

16 (Liertz, 2007; Thomas, Lane \& Kingston, 2011) might be necessary as self-confidence affects

17 perceptions of anxiety and control (Kenny, 2011; Lehrer, 1987). In line with the suggested

18 biopsychosocial approach, MPA might be addressed by Hanin's (1997) Individual Zone of

19 Optimal Functioning model which accounts for individual differences in the emotion-

20 performance relationship (as was explored by Osborne, 2016a and Osborne et al., 2014).

21 In similar fashion, Barron (1972) suggested that ego strength, defined as resilience, self-

22 control, adaptive coping and wellbeing, determines whether traits result in healthy or 


\section{Running Head: ENHANCEMENT WORK IN MUSIC}

1 pathological creativity (Nordin-Bates, 2012). Psychological training aimed at improving such

2 ego strength components can thus be crucial to musicians. For instance, Osborne (2013) found

3 that resilience training decreased MPA, failure avoidance, self-sabotage and disengagement and

4 improved self-belief, planning, persistence, and control over success in musicians.

$5 \quad$ Building psychologically resilient attitudes towards anxiety and practicing how to apply a

6 focus on task execution while distancing oneself from personal feelings of anxiety can be

7 another powerful tool to improve emotional communication and expression (Nordin-Bates,

8 2012). After all, "what do the performer's feelings of anxiety have to do with the emotion of the

9 piece that is to be conveyed?" Task-relevant focus is, hence, not a rejection of emotionality but

10 rather, a focus on task-appropriate emotionality which could be achieved via attentional control

11 training similar to those deployed in sports. The application of appropriate focus might be

12 especially relevant to musicians due to their tendency to focus "inward" and often on their

13 symptoms of MPA (Gill et al., 2006). Thus it might be worth teaching musicians the potential

14 benefits of applying an external focus (e.g., focusing on the effect of a movement rather than the

15 execution). External focus has shown similar benefits in both sports and music (Wulf, 2013).

16 Furthermore, attention allocation might be a mediator in experiences of MPA (Kageyama, 2007).

17 In addition, attentional focus can be used for the purposes of more effective motor skill

18 development in music (see Wulf \& Mornell, 2008). A holistic multisensory self-focus (see

19 Carson \& Collins, 2015) might also be explored to teach musicians on what and how to focus

20 their attention during an established skill. For instance, expert musicians have been found to

21 focus on thoughts related to physicality, confidence and task-relevant cognitions during

22 performance (Buma, Bakker \& Oudejans, 2014), alternating between conscious versus automatic 
1 and internal versus external focus, depending on need.

2 In addition, exploring the use of performance cues (Chaffin et al., 2009; Winter, MacPherson

3 \& Collins, 2014) mood words, temporally-structured cues (MacPherson, Collins \& Morriss,

4 2008), trigger words (Broomhead, Skidmore, Eggett \& Mills, 2012) and analogies might

5 resonate well with musicians as the concept of using words, metaphors and analogies (e.g.,

6 character markings in a piece) is already known to them. These cues and words could also

7 enhance emotional expression (Woody, 2002).

8 While most music performance is a closed task, with the exception of improvisation, decision-

9 making and error-management training could be used similarly to other disciplines involving

10 closed aesthetic tasks (Kruse-Weber \& Parncutt, 2014), especially in the context of

11 memorization.

12 Imagery is a commonly used strategy for various purposes in music, including the

13 development of emotional expression, psychological and physical skills (see e.g., Clark,

14 Williamon \& Aksentijevic, 2012). Although imagery use appears common, it is an undeveloped

15 skill in music (Haddon 2007). This might be addressed by teaching musicians how to use

16 advanced imagery models such as PETTLEP (Holmes \& Collins, 2001). PETTLEP is an

17 acronym in which each letter refers to a component that should be taken into consideration when

18 implementing imagery interventions. Respectively, the letters stand for: Physical, Environment,

19 Task, Timing, Learning, Emotion and Perspective. All these aspects should be considered in

20 order for the imagery exercise to resemble the performance situation as closely as possible. The

21 application of the PETTLEP model has already shown promise in musical settings (Folvig, 2011;

22 Wright, Wakefield \& Smith, 2014). 
1 When incorporating self-talk, however, it is important to match intensity to activity and

2 content (Hatzigeogiadis, Zourbanos \& Theodorakis, 2007) and also, the cultural context (Peters

$3 \&$ Williams, 2006). This is relevant as prestigious institutions draw performers from varying

4 cultural backgrounds, creating a second cultural layer to consider in addition to the more general

5 "western classical music" culture with its typical differences in goals and motivational

6 constructs.

7 As these possibilities suggest, there is much greater scope for exploration of transferrable

8 strategies than is currently deployed. Music performance is demanding enough to potentially

9 benefit from such a range of approaches available from sport psychology and practitioners

10 should therefore not be afraid to explore these within a holistic framework that considers the

11 communication of adaptive lifestyle habits, practice and performance preparation and

12 psychological skill development in a culturally-sensitive manner.

13

14

15 The domain of music performance is rife with various psychological, physical and

16 institutional challenges for which adequate support is limited or in its early stages of

17 development. Furthermore, performance psychology as it is used in sports for the purpose of

18 performance optimization is not widely known to musicians. Hence, performance psychologists

19 are increasingly being employed to work within a domain that shares similarities but also

20 exhibits key differences to sports. The impact of music's unique socio-cultural environment may

21 pose challenges that are worth considering when planning successful interventions. Issues

22 pertaining to divergences between sports and music such as fine motor skill development,

23 memorization, artistry, identity, coping styles, goals, motivational constructs, lifestyle habits, and 
1 perceptions of MPA are some of the prevalent areas that might be consequential. Accordingly,

2 future research might explore musicians' underlying beliefs related to dysfunctional behaviors as

3 well as the gaining of positive control and experiencing of positive change. In addition, the

4 extent to which positive behaviors are valued and whether and to what extent musicians would

5 commit to participating in progressive initiatives might render valuable knowledge.

6 Considering the implications of such challenges, this paper proposed deploying a culturally-

7 informed holistic approach to music performance psychology training that incorporates a range

8 of training methods and takes into account lifestyle habits, psychological skill development and

9 explores the appropriate phrasing and communication of such knowledge. We hope this

10 information might be of use to practitioners when communicating, translating and optimizing

11 existing performance enhancement methods from the sports domain to music performance. We

12 invite further research and discussion on the topic should the views presented be deemed

13 inaccurate or incomplete.

14

15

16

17

18

19

20

21 
Running Head: ENHANCEMENT WORK IN MUSIC

1

2

3

4

5

6 Allen, S. E. (2013). Memory stabilization and enhancement following music practice.

$7 \quad$ Psychology of Music, 41(6), 794-803.

8 Araújo, M. V. (2015). Measuring self-regulated practice behaviours in highly skilled musicians.

$9 \quad$ Psychology of Music. http://dx.doi.org/10.1177/0305735614567554

10 Ashford, K., \& Jackson, R. C. (2010). Priming as a means of preventing skill failure under

11 pressure. Journal of Sport Exercise Psychology, 32(4), 518-36.

12 Atkins, L. (2009). Health and wellbeing education in British conservatoires. In A. Williamon, S.

13 Pretty, \& R. Buck (Eds.), Proceedings of the International Symposium on Performance

14 Science 2009 (pp. 219-223). EAC.

15 Auer, L. (2003). Violin playing as I teach it. New York: Barnes \& Noble Publishing.

16 Barron, F. (1972). Artists in the making. New York: Seminar Press.

17 Baughman, M. M. (2014). An examination of methods used to teach practice strategies in the

18 college voice studio (Doctoral dissertation, University of Missouri, Columbia).

19 Bejjani, F. J., Kaye, G. M., \& Benham, M. (1996). Musculoskeletal and neuromuscular

20 conditions of instrumental musicians. Archives of Physical Medicine and Rehabilitation,

21 77(4), 406-413.

22 Bellon, D. (2006). Application of sport psychology to music performance: a study based on a

23 review of sport psychology literature and selected interviews with professional musicians 
1 (Doctoral Dissertation, Arizona State University, Ann Arbor).

2 Bennett, D., \& Stanberg, A. (2006). Musicians as teachers: developing a positive view through

3 collaborative learning partnerships. International Journal of Music Education, 24(3), 219-230.

4 Bernardi, N. F., Schories, A., Jabusch, H. C., Colombo, B., \& Altenmueller, E. (2013). Mental

5 practice in music memorization: an ecological-empirical study. Music Perception: An

6 Interdisciplinary Journal, 30(3), 275-290.

7 Bonneville-Roussy, A., \& Bouffard, T. (2015). When quantity is not enough: Disentangling the

8 roles of practice time, self-regulation and deliberate practice in musical achievement.

$9 \quad$ Psychology of Music, 43(5), 686-704. http://dx.doi.org/10.1177/0305735614534910

10 Braden, A. M., Osborne, M. S., \& Wilson, S. J. (2015). Psychological intervention reduces self-

11 reported performance anxiety in high school music students. Frontiers in Psychology, 6.

12 Brandon C. \& Ivens C. (2009). Thinking Skills for Peak Performance. South Yarra, Australia:

13 Macmillan Education Australia.

14 Broomhead, P., Skidmore, J. B., Eggett, D. L., \& Mills, M. M. (2012). The effects of a positive

15 mindset trigger word pre-performance routine on the expressive performance of junior high

16 age singers. Journal of Research in Music Education, 60(1), 62-80.

17 Brown, J. (2012). Making it relevant: engaging performing arts students in theoretical learning.

18 Educating Professional Musicians in a Global Context, 26.

19 Buma, L. A., Bakker, F. C., \& Oudejans, R. R. (2014). Exploring the thoughts and focus of

20 attention of elite musicians under pressure. Psychology of Music.

21 http://dx.doi.org/10.1177/0305735613517285

22 Burke, M. (2001). Obeying Until It Hurts: Coach-Athlete Relationships, Journal of the 
1 Philosophy of Sport, 28(2), 227-240.

2 Byo, J. L., \& Cassidy, J. W. (2008). An exploratory study of time use in the practice of music

3 majors self-report and observation analysis. Update: Applications of Research in Music

$4 \quad$ Education, 27(1), 33-40.

5 Carey, G., (2010). Performance or Learning? Reflections of pedagogical practices within the

6 conservatoire. In M. Hannan (Ed.) The Musician in Creative and Educational Spaces in the

721 st Century. Proceedings from the 18th International Seminar of the CEPROM.

8 Carey, G., \& Grant, C. (2014). Teachers of instruments, or teachers as instruments? From transfer

9 to transformative approaches to one-to-one pedagogy. Paper presented at the CEPROM

10 Seminar, Belo Horizonte, Brazil.

11 Carson, H.J \& Collins, D. (2015). The fourth dimension: A motoric perspective on the anxiety-

12 performance relationship. International Review of Sports and Exercise Psychology.

13 http://dx.doi.org/ 10.1080/1750984X.2015.1072231

14 Chaffin, R., \& Logan, T. (2006). Practicing perfection: How concert soloists prepare for

15 performance. Advances in cognitive psychology, 2(2-3), 113-130.

16 Chaffin, R., Demos, A., \& Crawford, M. (2009, December). Sources of variation in musicians'

17 use of performance cues. The $2^{\text {nd }}$ International Conference on Music Communication Science,

18 Sydney, Australia.

19 Chan, C., \& Ackermann, B. (2014). Evidence-informed physical therapy management of

20 performance-related musculoskeletal disorders in musicians. Frontiers in Psychology, 5.

21 Chesky, K. S., \& Hipple, J. (1999). Musicians' perceptions of widespread drug use among

22 musicians. Medical Problems of Performing Artists, 14, 187-195. 
1 Chesky, K., Dawson, W., \& Manchester,R. (2006). Health promotion in schools of music: Initial

2 recommendations for schools of music. Medical Problems of Performing Artists, 21, 142-144.

3 Clark, T., \& Williamon, A. (2011). Evaluation of a mental skills training program for musicians.

$4 \quad$ Journal of Applied Sport Psychology, 23(3), 342-359.

5 Clark, T., Holmes, P., \& Redding, E. (2011). Investigating the physiological demands of musical

6 performance. International Symposium on Performance Science, Toronto, Canada.

7 Clark, T., Williamon, A., \& Aksentijevic, A. (2012). Musical imagery and imagination: The

8 function, measurement and application of imagery skills for performance. In D.J. Hargreaves,

9 D.E. Miell \& R.A.R. MacDonald (Eds.) Musical imaginations, Oxford: Oxford UP.

10 Collins, D. (2013). The road to success: rocky, yellow-brick or paved with good intentions?

11 In Scharz, M. (Ed.) Foundations for Excellence Conference: Nurturing and supporting

12 talented young dancers and musicians, Totnes, UK.

13 Collins, D. (2014). Talent Development Environments: Optimising Output [Powerpoint Slides]

14 https://www.englandgolf.org/shared/get-file.ashx?id=8494\&itemtype=document.

15 Collins, D., \& Kamin, S. (2012). The performance coach. Handbook of sport and performance

16 psychology, 692-706.

17 Connolly, C., \& Williamon, A. (2004). Mental skills training. In A. Williamon (Ed.), Musical

18 excellence: Strategies and techniques to enhance performance (pp. 221-245). Oxford: Oxford

19 University Press.

20 Davis, M. (2005, September 30). John Williams. Retrieved July 30, 2015.

21 Douglas, T. (2007). Enhancement in sport, and enhancement outside sport. Studies in Ethics,

22 Law, and Technology, 1(1). 
1 Duckworth, A. L., Peterson, C., Matthews, M. D., \& Kelly, D. R. (2007). Grit, perseverance and

2 passion for long-term goals. Journal of Personality and Social Psychology, 92(6), 1087.

3 Duke, R. A., Simmons, A. L., \& Cash, C. D. (2009). It's not how much; it's how characteristics of

4 practice behavior and retention of performance skills. Journal of Research in Music

$5 \quad$ Education, 56(4), 310-321.

6 Dunkel, S. E. (1990). The audition process: Anxiety management and coping strategies.

7 Stuyvesant, NH: Pendragon Press.

8 Dweck, C. (2006). Mindset: The new psychology of success. NY: Random House.

9 Ericsson, K. A., Krampe, R. T., \& Tesch-Römer, C. (1993). The role of deliberate practice in the 10 acquisition of expert performance. Psychological Review, 100(3), 363.

11 Evans, P. (2015). Self-determination theory: An approach to motivation in music education.

12 Musicae Scientiae, Online first, 1-19. http://dx.doi.org/10.1177/1029864914568044

13 Fishbein, M., Middlestadt, S. E., Ottati, V., Straus, S., \& Ellis, A. (1988). Medical problems

14 among ICSOM musicians: overview of a national survey. Medical Problems of Performing $15 \quad$ Artists, 3(1), 1-8.

16 Fry H. J. (1986). Overuse syndrome of the upper limb in musicians. Medical Journal of $17 \quad$ Australia, 144(4) 182-3, 185.

18 Gaunt, H. (2007). One-to-one tuition in a conservatoire: the perceptions of instrumental and 19 vocal teachers. Psychology of Music, 36(1), 1-31. 
1 Gill, A., Murphy, F., \& Rickard, N. S. (2006). A preliminary examination of the roles of

2 perceived control, cortisol and perceptions of anxiety in music performance. Australian

3 Journal of Music Education, 1, 32-47.

4 Ginsborg, J. (2004). Strategies for memorizing music. In A. Williamon (Ed.) Musical excellence:

5 Strategies and techniques to enhance performance, 123-141.

6 Greene, D. (2002). Performance Success. New York, NY: Routledge, 17.

7 Greene, D. (2012a). 11 strategies for audition and performance success: a workbook for

8 musicians. http://psi.dongreene.com/. Accessed 10 December 2015.

9 Greene, D. (2013). The performance skills inventory. http://psi.dongreene.com/. Accessed 10

10 December 2015.

11 Guptill, C., Zaza, C., \& Paul, S. (2000). An occupational study of physical playing-related

12 injuries in college. Medical Problems of Performing Artists, 15(2), 86.

13 Haddon, E. (2007). What does mental imagery mean to university music students and their

14 professors. In Proceedings of the ISPS. (pp. 301-306). Porto, Portugal.

15 Hanin, Y. L. (1997). Emotion and athletic performance: Individual zones of optimal functioning

16 model. European Yearbook of Sport Psychology, 1, 29-72.

17 Hatzigeorgiadis, A., Zourbanos, N., \& Theodorakis, Y. (2007). The moderating effects of self-

18 talk content on self-talk functions. Journal of Applied Sport Psychology, 19(2), 240-251.

19 Hawkes, M. (2015, February). Enhancing performance: a study of performance coaching in

20 practice in a UK conservatoire. Paper presented at the Reflective Conservatoire Conference, 21 London, UK.

22 Hays, K. F. (2002). The enhancement of performance excellence among performing artists. 
1 Journal of Applied Sport Psychology, 14(4), 299-312.

2 Hays, K. F. (2012). The Psychology of Performance in Sport and Other Domains. The Oxford

3 Handbook of Sport and Performance Psychology, 24.

4 Hays, K. F., \& Brown, C. H. (2004). You're on! Consulting for peak performance. Washington,

5 DC: American Psychological Association.

6 Holliday, B., Burton, D., Sun, G., Hammermeister, J., Naylor, S., \& Freigang, D. (2008).

7 Building the better mental training mousetrap: Is periodization a more systematic approach to

8 promoting performance excellence? Journal of Applied Sport Psychology, 20(2), 199-219.

9 Holmes, P. S., \& Collins, D. J. (2001). The PETTLEP approach to motor imagery: A functional

10 equivalence model for sport psychologists. Journal of Applied Sport Psychology, 13(1), 60-

1183.

12 Huhtanen, K. (2004). Once I had a promising future (facing reality as an ex-promising pianist).

13 Australian Music Forum, 10(3), 21-27.

14 Ivaldi, A., \& O'Neill, S. A. Motivation and identity in music performance: an exploratory study.

15 In C. Woods, G. B. Luck, R. Brochard, F. Seddon, \& J. A. Sloboda (Eds.), Proceedings of the

$16 \quad 6^{\text {th }}$ International Conference on Music Perception and Cognition. Keele University, UK.

17 Janzen, T. B., Thompson, W. F., Ammirante, P., \& Ranvaud, R. (2014). Timing skills and

18 expertise: discrete and continuous timed movements among musicians and athletes. Frontiers 19 in Psychology, 5. 
1 Juncos, D. G., \& Markman, E. J. (2015). Acceptance and Commitment Therapy for the treatment

2 of music performance anxiety: A single subject design with a university student. Psychology

3 of Music. http://dx.doi.org/0305735615596236.

4 Kageyama, NJ (2007). Attentional Focus as a Mediator in the Anxiety-Performance

5 Relationship: The Enhancement of Music Performance Quality Under Stress. (Doctoral

6 Dissertation, Indiana University, Indiana).

7 Kemp, A. E. (1996). The musical temperament. Psychology and personality of musicians.

8 Oxford: Oxford UP.

9 Kenny, D. (2011). The psychology of music performance anxiety. Oxford UP.

10 Kenny, D., Ackermann, B. (2009). Optimizing physical and psychological health in performing

11 musicians. In S. Hallam, I. Cross, \& M. Thaut (Eds.), Oxford Handbook of Music Psychology,

12 (pp. 390-400). Oxford: Oxford UP.

13 Kostka, M. J. (2002). Practice expectations and attitudes: A survey of college-level music

14 teachers and students. Journal of Research in Music Education, 50(2), 145-154.

15 Kruse-Weber, S. \& Parncutt, R. (2014) Error management for musicians: an interdisciplinary

16 conceptual framework. Frontiers in Psychology. 5, 777.

17 http://dx.doi.org/10.3389/fpsyg.2014.00777

18 Lacaille, N., Koestner, R., \& Gaudreau, P. (2007). On the value of intrinsic rather than traditional

19 achievement goals for performing artists: a short-term prospective study. International

20 Journal of Music Education, 25(3), 245-257.

21 Lacaille, N., Whipple, N., \& Koestner, R. (2005). Reevaluating the benefits of performance

22 goals: The relation of goal type to optimal performance for musicians and athletes. Medical 


\section{Running Head: ENHANCEMENT WORK IN MUSIC}

$1 \quad$ Problems of Performing Artists, 20(1), 11-16.

2 Lehrer P. M. (1987). A Review of the approaches to the management of tension and stage fright

$3 \quad$ in music performance. Journal of Research in Music Education, 35(3), 143-153.

4 Leinsdorf, E. (1999). Arthur Rubinstein. New York: RCA Red Seal.

5 Liertz, C. (2007). New Frameworks for Tertiary Music Education-A Holistic Approach for Many

6 Pyramids of Excellence. 8th Australasian Piano Pedagogy Conference. Australian National

$7 \quad$ University.

8 MacNamara, A. (2011). Psychological characteristics of developing excellence. In D. Collins, A.

9 Button \& H. Richards (Eds.), Performance psychology: A practitioner's guide, (pp. 47-64).

10 Oxford: Churchill Livingston.

11 MacNamara, A., \& Collins, D. (2009). More than the ' $X$ 'factor! A longitudinal investigation of

12 the psychological characteristics of developing excellence in musical development. Music

13 Education Research, 11(3), 377-392.

14 MacPherson, A. C., Collins, D., \& Morriss, C. (2008). Is what you think what you get?

15 Optimizing mental focus for technical performance. The Sport Psychologist, 22(3), 288-303.

16 Martindale, A., \& Collins, D. (2005). Professional judgment and decision making: The role of

17 intention for impact. Sport Psychologist, 19(3), 303.

18 McPherson, G. E., Evans, P., Kupers, E., \& Renwick, J. (2016). Applying self-determination and

19 self-regulation theories for optimising music performance. In A. Mornell (Ed). Art in Motion

20 III: Performing Under Pressure. Frankfurt, DE: Peter Lang.

21 Miksza, P. (2011). Relationships among achievement goal motivation, impulsivity, and the

22 music practice of collegiate brass and woodwind players. Psychology of Music, 39(1), 50-67. 
Running Head: ENHANCEMENT WORK IN MUSIC

1 Miksza, P. (2015). The effect of self-regulation instruction on the performance achievement,

2 musical self-efficacy, and practicing of advanced wind players. Psychology of Music, 43(2),

$3 \quad$ 219-243. http://dx.doi.org/10.1177/0305735613500832

4 Mills, J. (2006). Performing and teaching: The beliefs and experience of music students as

$5 \quad$ instrumental teachers. Psychology of Music, 34(3), 372-390.

6 Mishra, J. (2004). A Model of Musical memory. Proceedings of the 8th International Conference

7 on Music Perception and Cognition. Adelaide, Australia (pp. 74-86).

8 Mishra, J. (2011). Influence of strategy on memorization efficiency. Music Performance

9 Research, 4, 60-71.

10 Morgenstern, M. (2005). Physiological strain and emotional involvement: investigating heart

11 rate and heart rate variability of musical performers. Proceedings of the International

12 Conference on Psychological, Philosophical and Educational Issues in Musical Performance.

13 Porto, Portugal (p.175).

14 Mula, M., \& Trimble, M. R. (2009). Music and madness: Neuropsychiatric aspects of music.

$15 \quad$ Clinical medicine, 9(1), 83-86.

16 Nash, C., \& Collins, D. (2006). Tacit knowledge in expert coaching: Science or art? Quest, 58(4),

$17 \quad 465-477$.

18 Nordin-Bates, S. M. (2012). Performance Psychology in the Performing Arts. In S. Murphy

19 (Ed.), The Oxford Handbook of Sport and Performance Psychology (pp. 81-117). OUP.

20 Novick, J., \& Steen, R. (2014). Love is the drug: performance-enhancing in sport and music.

21 Sport in Society, 17(3), 419-432.

22 Olevsky, D. (2012). The study of success in music: Applying methods developed by sports 


\section{Running Head: ENHANCEMENT WORK IN MUSIC}

1 psychology towards achieving peak performance. (Doctoral Dissertation, University of

2 California, Santa Barbara).

3 Osborne, M. S. (2013). Maximising performance potential: the efficacy of a performance

$4 \quad$ psychology program to reduce music performance anxiety and build resilience in adolescents.

5 In A. Williamon, \& W. Goebl (Eds.), Proceedings of the International Symposium on

6 Performance Science (pp. 303-310). AEC.

7 Osborne, M. S. (2016a). Building performance confidence. In G. E. McPherson (Ed.), The child

8 as musician: A handbook of musical development (2nd ed., pp. 422-440). Oxford: Oxford UP.

9 Osborne, M. S., \& Patston, T. (2013, October 24-27). The developmental progression of music

10 performance anxiety and perfectionism: Implications for prevention and early intervention in

11 adolescent musicians. Paper presented at the Australian Association for Cognitive and

12 Behaviour Therapy 36th National Conference 'Innovations in Australasian Mental Health

13 Care', Hotel Grand Chancellor, Adelaide.

14 Osborne, M. S., Greene, D., \& Immel, D. (2014). Managing performance anxiety and improving

15 mental skills in conservatoire students through performance psychology training: a pilot

16 study. Psychology of Well-Being, 4(18), 1-17. http://dx.doi.org/10.1186/s13612-014-0018-3

17 Panebianco-Warrens, C. R., Fletcher, L., \& Kreutz, G. (2014). Health-promoting behaviors in

18 South African music students: A replication study. Psychology of Music.

19 http://dx.doi.org/10.1177/0305735614535829

20 Papageorgi, I., Creech, A., \& Welch, G. (2013). Perceived performance anxiety in advanced

21 musicians specializing in different musical genres. Psychology of Music, 4l(1), 18-41.

22 Parncutt, R. (2007). Can researchers help artists? Music performance research for music 


\section{Running Head: ENHANCEMENT WORK IN MUSIC}

students. Music Performance Research, 1(1), 1-25.

2 Parncutt, R., \& Williamon, A. (2005) Incorporating performance research into post-secondary

3 music curricula: Political and pedagogical strategies. Proceedings of the 2005 ESCOM

4 conference. Porto, Portugal.

5 Patston, T., \& Osborne, M. S. (2015). The developmental features of music performance anxiety

6 and perfectionism in school age music students. Performance Enhancement \& Health.

7 http://dx.doi.org/10.1016/j.peh.2015.09.003

8 Perkins, R. (2013). Learning cultures and the conservatoire: An ethnographically-informed case

$9 \quad$ study. Music Education Research, 15(2), 196-213.

10 Peters, H. J., \& Williams, J. M. (2006). Moving cultural background to the foreground: An

11 investigation of self-talk, performance, and persistence following feedback. Journal of

$12 \quad$ Applied Sport Psychology, 18(3), 240-253.

13 Petrides, K. V., Niven, L., \& Mouskounti, T. (2006). The trait emotional intelligence of ballet

14 dancers and musicians. Psicothema, 18(1), 101-107.

15 Pianist tells of adding sports psychology to his repertoire. (2014, February 26). Herald Scotland.

16 Retrieved July 30, 2015, from http://www.heraldscotland.com

17 Platz, F., Kopiez, R., Lehmann, A. C., \& Wolf, A. (2014). The influence of deliberate practice on

18 musical achievement: a meta-analysis. Frontiers in psychology, 5.

19 Poczwardowski, A., \& Conroy, D. E. (2002). Coping responses to failure and success among

20 elite athletes and performing artists. Journal of Applied Sport Psychology, 14(4), 313-329.

21 Quarrier, N. F. (1993). Performing arts medicine: the musical athlete. Journal of Orthopaedic \& 22 Sports Physical Therapy, 17(2), 90-95. 
1 Quarrier, N. F. (2013). Musicians are athletes too. Journal of Yoga \& Physical Therapy, 3, 141.

2 Ray, O. (2004). How the mind hurts and heals the body. American Psychologist, 59(1), 29.

3 Renshaw, P. (2004). Connecting Conversations: the changing voice of the artist. New Practices

$4 \quad$ New Pedagogies, 99-116.

5 Rickert, D. L., Barrett, M. S., \& Ackermann, B. J. (2015). Are music students fit to play? A case

6 study of health awareness and injury attitudes amongst tertiary student cellists. International

7 Journal of Music Education. http://dx.doi.org/10.1177/0255761415582343

8 Service, T. (2012). Melvyn Tan Turns Guinea Pig: Are Musicians Like Athletes? Do Concert

$9 \quad$ Pianists Need Olympian Levels Of Fitness To Cope With The Strains Of Performance?

10 Science Is Using Melvyn Tan To Find Out'. The Guardian, 2012. Web. 26 July 2015.

11 Shulman, L. S. (2005). Pedagogies. Liberal Education, 91(2), 18-25.

12 Simmons, A. L. (2012). Distributed practice and procedural memory consolidation in musicians' 13 skill learning. Journal of Research in Music Education, 59(4), 357-368.

14 Simmons, A. L., \& Duke, R. A. (2006). Effects of sleep on performance of a keyboard melody.

15 Journal of Research in Music Education, 54(3), 257-269.

16 Sinden, L. M. (1999). Music performance anxiety: Contributions of perfectionism, coping style, 17 self-efficacy, and self-esteem. (Doctoral Dissertation, Arizona State University, Arizona).

18 Steptoe, A. (1989). Stress, coping and stage fright in professional musicians. Psychology of $19 \quad$ Music, 17(1), 3-11.

20 Steyn, M. H. (2013). The impact of psychological skills and mindfulness training on the 21 psychological well-being of undergraduate music students (Doctoral dissertation, University 22 of Pretoria). 
1 Talbot-Honeck, C., \& Orlick, T. (1998). The essence of excellence: Mental skills of top classical

2 musicians. Journal of Excellence, 1, 61-75.

3 Taylor, A.H. \& Wasley, D. (2004). Physical fitness. In A. Williamon (ed.), Musical Excellence;

$4 \quad$ strategies and techniques to enhance performance. (pp. 163-178). Oxford: Oxford UP.

5 Thomas, O., Lane, A., \& Kingston, K. (2011). Defining and contextualizing robust sport-

6 confidence. Journal of Applied Sport Psychology, 23(2), 189-208.

7 Thomson, J. (2014). Training for performance: Lessons from sports psychology applied to

8 musical training (Doctoral dissertation, University of California, Los Angeles).

9 Tindall, B. (2004). Better playing through chemistry. New York Times, 17.

10 Wasley, D., Taylor, A., Backx, K., Williamon, A. (2012). Influence of fitness and physical

11 activity on cardiovascular reactivity to musical performance. Work. 41(1), 27-32.

12 Watson, A. H. (2009). The biology of musical performance and performance-related injury.

13 Lanham, MD: Scarecrow Press.

14 Welford, A. T. (1968). Fundamentals of skill. New York: Methuen

15 Weller, J (2004) The Whole Musician: Journey to authentic vocation. In O. Musumeci (Ed.), The

16 ISME Commission for the Education of the Musician. Preparing musicians: Making sound

17 worlds (pp. 245-256). Barcelona: Generalitat de Catalunya Department d'Ensenyament.

18 Weller, J. (2008). Creating a life in music: Theory to praxis. In D. Bennett \& M. Hannan (Eds.),

19 Inside, outside, downside up: Conservatoire training and musicians' work (pp. 149-158).

20 Perth: Black Swan Press.

21 Weller, J. (2010, August 1-6). Transitioning to Professional Life. Paper presented at the $29^{\text {th }}$

22 ISME World Conference, Beijing. 
1 Wesner, R. B., Noyes, R., \& Davis, T. L. (1990). The occurence of performance anxiety among 2 musicians. Journal of Affective Disorders, 18(3), 177-185.

3 Wilkinson, D. (2005). We've All Been There: Attitudes of Music Conservatoire Staff Towards

4 Performance Anxiety, and Possible Implications for Students (Doctoral dissertation,

5 University of Sheffield, UK).

6 Williamon, A. (1999). The value of performing from memory. Psychology of Music, 27(1), 84-

795.

8 Williamon, A., \& Thompson, S. (2006). Awareness and incidence of health problems among

9 conservatoire students. Psychology of Music, 34(4), 411-430.

10 Williamon, A., \& Valentine, E. (2000). Quantity and quality of musical practice as predictors of 11 performance quality. British Journal of Psychology, 91(3), 353-376.

12 Williamon, A., Aufegger, L., \& Eiholzer, H. (2014). Simulating and stimulating performance:

13 introducing distributed simulation to enhance musical learning and performance. Frontiers in

14 Psychology, 5(25), 1-9. http://dx.doi.org/10.3389/fpsyg.2014.00025

15 Willmott, T. \& Collins, D. (2015). Challenges in the Transition to Mainstream: Promoting

16 Progress and Minimizing Injury in Freeskiing and Snowboarding. Sport in Society.

17 http://dx.doi.org/10.1080/17430437.2015.1031530

18 Winter, S., \& Collins, D. (2015). Why Do We Do, What We Do? Journal of Applied Sport

19 Psychology, 27(1), 35-51.

20 Winter, S., MacPherson, A. C., \& Collins, D. (2014). "To think, or not to think, that is the 21 question”. Sport, Exercise, and Performance Psychology, 3(2), 102.

22 Wolfe, M. L. (1990). Coping with musical performance anxiety: Problem-focused and emotion- 
1 focused strategies. Medical Problems of Performing Artists, 5(1), 33-36.

2 Woody, R. H. (2002). Emotion, imagery and metaphor in the acquisition of musical performance

$3 \quad$ skill. Music Education Research, 4(2), 213-224.

4 Wright, D. J., Wakefield, C. J., \& Smith, D. (2014). Using PETTLEP imagery to improve music

5 performance: A review. Musicae Scientiae, 18(4), 448-463.

6 Wulf, G. (2013). Attentional focus and motor learning: A review of 15 years. International

$7 \quad$ Review of Sport and Exercise Psychology, 6(1), 77-104.

8 Wulf, G., \& Mornell, A. (2008). Insights about practice from the perspective of motor learning: a 9 review. Music Performance Research, 2, 1-25.

10 Zenker, R. (2004). Music as a Lifelong Pursuit: Educating for a Musical Life. In L.R. Bartel, 11 (Ed.) Questioning the Music Education Paradigm (pp. 121-135). Canadian Music Educators' 12 Association. 